\title{
Karol Karp \\ (Toruń) \\ IL CONFLITTO TRA INDIVIDUO E SISTEMA: \\ VERSO LA COSTRUZIONE DELLA NUOVA \\ IDENTITÀ CULTURALE NEL ROMANZO \\ „I GRANDI OCCHI DEL MARE” DI LEONARD GUACI
}

\begin{abstract}
The aim of the article is to analyse the theme of cultural identity in the novel „I grandi occhi del mare” by Leonard Guaci. Starting from Gioia di Cristofaro Longo's theoretical assumptions concerning the connection between identity and culture, we stress the influence of Italian culture on the sense of identity felt by the characters who represent Albanians living in the period of Enver Hoxha's communist dictatorship. The analysis also draws on the theories of scholars such us Hannah Arendt, Homi Bhabha, Dominique Chancé, Giovanni Marchetti, Nora Moll.
\end{abstract}

\section{Key words}

Guaci, dictatorship in Albania, Italian television, Italian culture, cultural identity 


\section{INTRODUZIONE}

Secondo Gioia di Cristofaro Longo „l'identità (...) è la forma che la cultura, intesa come patrimonio di idee, valori, norme, orientamenti, assume dal momento in cui, in seguito al processo di inculturazione e socializzazione, entra a far parte del sistema culturale di riferimento del soggetto"1. La studiosa giustamente evidenzia l'esistenza di uno stretto legame tra cultura e identità che sul piano ontologico risultano nozioni sinonimiche, accenna anche alla loro reciproca dipendenza:

l'identità è (...) un principio cognitivo operativo e regolativo, per il quale un soggetto si orienta all'azione e sceglie tra più alternative possibili preservando la sua coerenza psichica e culturale. Alternative che si dipanano tra rotture e resistenze attraverso le quali la cultura insieme all'uomo procede ${ }^{2}$.

La cultura forgia l'identità, ma al contempo è completamente condizionata dall'individuo dotato della capacità di operare una scelta libera tra gli spazi culturali a cui desidera appartenere. Cristofaro Longo nota che paradossalmente la libertà che lo caratterizza può dare luogo a situazioni conflittuali e „si tratta di quei casi in cui individui o gruppi affermano valori che sono oggettivamente in contrasto con i valori espressi dalla classe dominante"3. Tale stato di cose spicca nel secondo romanzo di Leonard Guaci (1967), uno scrittore migrante d'espressione italiana e origine albanese, intitolato „I grandi occhi del mare" (2005), che intendiamo qui indagare per determinare l'influenza della cultura italiana sul senso di appartenenza identitaria dei personaggi principali.

\section{ZYZ}

La strategia narrativa adottata da Guaci è imperniata su due dimensioni che si compenetrano. Da un lato lo scrittore si concentra sulla descrizione di una famiglia, sulle relazioni tra i suoi membri, studiando la loro autocoscienza, dall'altro lato delinea un'ampia immagine dell'Albania nell'epoca della dittatura di Enver Hoxha ${ }^{4}$. Il suo romanzo, munito di una lingua scorrevole e vivace che mira alla semplicità sintassica e chiarezza dell'informazione

${ }^{1}$ G. Di Cristofaro Longo, Identità e cultura. Per un'antropologia della reciprocità, Roma 1993, p. 34.

${ }^{2}$ Ibidem, p. 36.

${ }^{3}$ Ibidem, p. 26.

${ }^{4}$ Occorre precisare che la trama ingloba la storia dell'Albania che va dalla fine della seconda guerra mondiale agli anni Novanta del Novecento. 
trasmessa, mescola vari elementi tipicamente letterari con quelli socioculturali. Tale tendenza è generalmente presente in altri autori migranti, non di rado desiderosi di parlare del proprio paese d'origine.

L'opera, suddivisa in quattordici capitoli, è ambientata a Valona, una città albanese affacciata sul Mar Adriatico. Il lettore si immerge nella trama attraverso la narrazione in terza persona che, vista la sua specificità, dovrebbe creare una distanza significativa tra il narratore, i protagonisti e il lettore. In Guaci però il narratore sembra simpatizzare con protagonisti precisi modellando anche l'atteggiamento del lettore, invitandolo a formulare giudizi sugli avvenimenti in cui sono coinvolti. Abbiamo a che fare dunque con una narrazione extradiegetica particolare in quanto in un certo senso essa funge da ponte tra il fruitore dell'opera e i suoi principali elementi costitutivi. Il tono del romanzo è prevalentemente neutro, comunque in alcune parti diventa solenne. Si tratta delle parti in cui la prosa di Guaci è evidentemente impegnata. L'autore intende mettere in risalto l'importanza che dà ad alcune vicende descritte, per esempio al crollo della dittatura comunista, e ciò risulta senz'altro dalla sua biografia.

Nell'opera in esame campeggiano i meccanismi usati dal regime per controllare i membri della società: la polizia segreta Sigurimi e numerose spie fanno di tutto per eliminare eventuali atteggiamenti reazionari. La vita degli albanesi corre all'insegna di un costante timore di non essere considerati nemici del sistema in quanto ciò significherebbe una lunga carcerazione oppure la morte. Ci sono però degli individui che decidono di infrangere le regole dettate dal comunismo, tra i quali emerge il personaggio di Zyz, un uomo maturo che da giovane ha vissuto parecchi anni in Italia, godendo di una libertà illimitata. Abbandonati gli studi, è divenuto musicista per sviluppare la passione per la materia che lo interessava da sempre. Il protagonista possiede un sapere rilevante sui vari elementi culturali della civiltà italiana, quelli inerenti all'arte, alla storia, allo stile di vita degli abitanti della penisola. Dopo il ritorno in Albania ha nostalgia dell'Italia e, se potesse, non esiterebbe a giungervi subito.

In realtà, confessò, aveva sognato mille volte di ritornarci. Avrebbe voluto rivisitare
tutti i luoghi dove aveva suonato, i locali dove si era ubriacato insieme alla sua
donna e gli alberghi dove aveva dormito. Esprimeva quel desiderio come un uomo
cosciente di chiedere la cosa più impossibile del mondo. (...) valanghe di ricordi lo
assillavano come voci invitanti di sirene marine. Si contorceva tutto come sotto
l'effetto di spettri luminosi, cadeva preda di molti tic nervosi, sembrando ancora
più pazzo di quanto lo credessero, ma poi accendeva l'ennesima sigaretta e con
le nuvolette di fumo scacciava fuori anche l'agitazione ${ }^{5}$.

${ }^{5}$ L. Guaci, I grandi occhi del mare, Nardò 2005, pp. 54-55. 
Visto il carattere oppressivo del sistema comunista che vieta contatti con l'Occidente, Zyz è consapevole dell'impossibilità di recarsi in Italia. Così, sebbene si esponga al rischio di perdere la vita, crea intorno a sé una dimensione imbevuta di italianità e di spirito occidentale, ne diventa divulgatore non solo per soddisfare il bisogno di sentirsi più vicino ai tempi felici, ma esprime anche il suo disappunto verso la politica delle autorità, che fanno dell'Albania una gabbia ben chiusa, la isolano dal resto del mondo e ne ostacolano lo sviluppo.

La casa del protagonista, in cui è riuscito a raccogliere molti libri, opere dei più famosi e apprezzati scrittori provenienti da tutto il mondo, acquisisce un carattere particolare, si presenta come un'oasi di libertà, di lotta contro l'inerzia che caratterizza la maggior parte della società albanese, sempre fedele al dittatore. Della possibilità di sprofondarsi nella lettura è meravigliata Aulona, l'unica femmina dei dodici figli dei coniugi Cipi, Arta e Viron, la quale Zyz introduce nell'atmosfera del suo regno. In un breve periodo di tempo tra loro si instaura un rapporto di amicizia e fedeltà; nei loro cuori nasce la passione per la musica e il desiderio di (ri)scoprire il paese sull'altra sponda dell'Adriatico. Secondo Aulona li accosta anche l'atteggiamento che gli altri hanno nei loro confronti:

Nelle parole di Zyz trovava anche un po' se stessa. Le sue fughe, i suoi interminabili silenzi, le lunghe ore di solitudine sulla spiaggia ora avevano un nome: libertà. Come Zyz, anche lei in mezzo ai suoi coetanei era giudicata „strana”. Le compagne di scuola non la trattavano come una di loro, si allontavano come per timore che le contagiasse. (...) Anche le insegnanti si rassegnavano davanti ai suoi modi, chi per paura, chi per incapacità e non erano mai state disposte a capirla. Ma era ciò che lei voleva, non essere compresa, essere messa da parte (...) era qualcosa che veniva decretato e ordinato dall'inconscio e, quindi, non programmabile ${ }^{6}$.

I protagonisti vivono isolati dagli altri, incompresi e ritenuti strani, anzi pazzi, ma ciò consente loro di sentirsi più liberi in quanto si immergono in una dimensione costruita da loro stessi e imperniata sui pilastri che scelgono da soli. Zyz però non si considera pazzo, come mette in risalto lui stesso:

I veri pazzi sono gli altri, quelli che sembrano normali. Loro hanno le maschere. Il mio volto è nudo, perché la luce lo possa baciare. La mia anima avampa d'amore per il sole. Essere pazzo è un modo per gustare la mia libertà, per spaventare chi me ne vuole privare"7.

Secondo Zyz il concetto di pazzia risulta inseparabilmente legato al portare una maschera. Ai propri occhi il protagonista è nudo, cioè vero, invece gli

${ }^{6}$ Ibidem, p. 64.

${ }^{7}$ Ibidem, p. 63. 
altri sono falsi, mascherati e non riescono a esternare il loro ego. Nel suo immaginario mascherarsi significa essere fedeli al regime, vivere condizionati dalle sue impostazioni, limitare la propria libertà. Chi decide di farlo, si accosta in modo estremo alla follia. Lui dunque non può essere pazzo perché non si sottomette alle regole del sistema.

Zyz si nutre di ricordi, sovente gli riaffiora alla mente l'immagine dell'Italia. Aulona invece trascorre molto tempo in solitudine, ammira il mare. Nel romanzo in esame il mare si distingue per una doppia natura: da un lato costituisce una barriera, un ostacolo che va sormontato per raggiungere lo scopo prefissato, dall'altro si dimostra come l'unica possibilità di fuga verso una dimensione migliore. I protagonisti sono costretti ad attraversarlo, a intraprendere un lungo viaggio per giungere in Italia, luogo dello loro salvezza, dove possono sperare bene per il futuro ${ }^{8}$.

Aulona sovente varca l'orizzonte con i pensieri, curiosa della realtà che le pare tanto lontana, ma a cui si avvicinerà proprio attraverso gli incontri con Zyz.

Zyz le parlava molto dell'Italia. Non solo della sua musica ma anche della sua storia, della guerra, di Gramsci, Mussolini, Verdi, Leopardi, Garibaldi. A volte si spingeva molto più indietro, fino a Marco Aurelio o Giulio Cesare. Quando parlava stendeva lo sguardo in avanti come un marinaio, gli occhi diventavano piccoli come noccioline e il petto si apriva come una fisarmonica. Era la sua seconda patria e gli mancava tanto?

Agli occhi della protagonista Zyz si rivela un vero e proprio maestro che stuzzica al massimo la sua immaginazione, le consente di conoscere meglio il mondo e di sviluppare il talento musicale ${ }^{10}$. Dopo l'uccisione dell'uomo da parte degli agenti della dittatura, la ragazza si crede depositaria del suo retaggio mentale, si cura dei suoi libri e intende trasformare la sua casa in biblioteca affinché possano farne uso tutti gli appassionati di lettura. Ciò non si rivela possibile che dopo il crollo del regime. I libri di Zyz sono raccolti in una sala messa a disposizione dal comune di Valona. Viene creata una biblioteca che simboleggia il fallimento della politica del comunismo, ossia la vittoria della giustizia, il rispetto delle leggi che spettano a ogni individuo. La biblioteca è curata da Viron. Il fatto risulta molto importante in quanto marca l'ultima tappa della trasformzione avvenuta nella sua personalità. Nelle

\footnotetext{
${ }^{8}$ Una tipologia simile del motivo si percepisce in altri autori in lingua italiana di origine albanese. A proposito si vedano: „Il mosaico del tempo grande” (2012) di Carmine Abate e „I nipoti di Scanderbeg" (2012) di Artur Spanjolli.

${ }^{9}$ L. Guaci, I grandi occhi del mare, p. 54.

${ }^{10} \mathrm{La}$ ragazza suona la chitarra e canta.
} 
parti iniziali del romanzo l'uomo, l'ex-partigiano e un grande patriota, si impegna con zelo nel supporto della dittatura. Una volta arrestato per futili motivi, del resto falsi, scopre sulla propria pelle l'ingiustizia che la caratterizza. Il volto negativo del sistema diventa per lui ancora più visibile quando analizza i meccanismi che esso usa per controllare la società e le conseguenze subite dai suoi figli. L'uomo teme per loro, comunque non può manifestare espressamente il suo atteggiamento verso le autorità. In fin dei conti la sua inerzia si trasforma in azione e proprio la biblioteca ne costituisce una buona prova.

Aulona risulta così legata a Zyz da essere sicura che il suo fantasma la accompagni, ogni tanto ha l'impressione di parlare con lui. Il suo atteggiamento potrebbe essere visto come l'intenzione di agire conformemente ai valori accettati in precedenza e sempre ritenuti giusti, come il proposito di mantenere la parola, il quale, secondo Paul Ricoeur ${ }^{11}$, è uno dei fattori che caratterizzano l'identità dell'individuo. In base alla teoria dello studioso pare possibile azzardare l'ipotesi che Aulona inizi ad acquisire la nuova identità culturale proprio a contatto con Zyz.

\section{LA TELEVISIONE E LA RADIO}

Il maestro, rendendosi conto che le autorità albanesi non rispettano le basilari leggi dell'uomo, fa uno sforzo strenuo per propagare l'italianità e lo spirito occidentale, sperando probabilmente che la situazione cambi e l'Albania segua in qualche modo il modello socio-culturale diffuso in Italia. Influenzata da lui, Aulona è sempre più desiderosa di avvicinarsi al mondo che esiste dall'altra parte del mare. Visti l'isolamento del paese e l'impossibilità di passare il confine, il mezzo perfetto per realizzarlo si dimostrano la televisione e la radio. I programmi portatori di idee capitaliste sono proibiti ma la curiosità della ragazza e la fedeltà agli ideali trasmessi da Zyz la spingono a rischiare. Così viene a conoscenza dei fatti inerenti a varie sfere della vita, che per molti dei suoi connazionali rimangono inimmaginabili. Al centro del suo interesse si trova la cultura italiana e soprattutto la musica, scopre il fascino del festival di Sanremo, si innamora delle canzoni belle e melodiose di artisti come Adriano Celentano, Lucio Battisti o Eros Ramazzotti. Guardare la televisione diventa una sorta di rito, un'azione ciclica e solenne che Aulona compie

\footnotetext{
${ }^{11}$ P. Ricoeur, Filozofia osoby, Kraków 1992, p. 34.
} 
insieme a quasi tutti i suoi fratelli per essere in fin dei conti propensa a percepire la realtà attraverso i messaggi che crea, la parola orale che favorisce ${ }^{12}$.

Come evidenzia Nora Moll,

non sono solo la cultura popolare e la politica nazionale italiane a entrare nell'immaginario collettivo albanese attraverso la Tv: essa infatti funge anche da filtro per il mondo occidentale, narrando quegli aspetti dell'attualità politica e culturale internazionale offuscati dalle narrazioni ufficiali del Paese delle aquile ${ }^{13}$.

Commentando l'influenza della televisione italiana sui protagonisti di Guaci, la studiosa nota anche che in alcuni casi risulta difficile interpretare le informazioni trasmesse:

Va sottolineato che degli aspetti dell'autoimmagine italiana riverberata dalla Rai, lo scrittore albanese approfondisce sempre e comunque gli effetti sulle coscienze dei suoi personaggi principali, effetti che ben presto mostrano la loro intrinseca ambiguità. Infatti i fratelli Cipi fanno fatica a decifrare gli avvenimenti che caratterizzano la crisi politica italiana degli anni '70: appoggiano incondizionatamente i partiti conservatori italiani e non comprendono perché dei terroristi comunisti si impegnano in una lotta armata per distruggere il loro Eden, fatto di canzoni di Sanremo, di presentazioni attraenti e intelligenti, di campionati di calcio e di film d'amore ${ }^{14}$.

Moll giustamente osserva che a volte il materiale socio-culturale su cui indaga Guaci sarebbe più facile da interpretare per il destinatario italiano che per quello albanese in quanto il primo, attraverso la memoria, riesce a identificarsi con alcuni suoi elementi:

(...) la lettura de „I grandi occhi del mare” (...) si configura come uno stimolo costante della memoria, una memoria culturale sentita anch'essa come „privata”, indivisibile e comprensibile solo da chi è cresciuto in seno alla società narrata dalla televisione e dalla radio ${ }^{15}$.

In un certo senso guardare la televisione e opporsi al regime significano seguire l'esempio di Zyz e continuare insieme ad Aulona la sua missione. Vale la pena mettere in evidenza che il motivo della televisione nella società dell'Albania nell'epoca del comunismo appare anche nel romanzo „Cronaca di una vita in silenzio" (2003) di Artur Spanjolli. La sua tipologia risulta ben diversa da quella nell'opera in esame. Spanjolli non si concentra sul ruolo che la televisione riveste nella costruzione dell'identità dei protagonisti, ma rileva

\footnotetext{
${ }^{12}$ Sul ruolo della parola orale cfr. W.J. Ong, Oralità e scrittura. La tecnologia della parola, Bologna 2006, pp. 90-91.

${ }^{13} \mathrm{~N}$. Moll, Il ruolo della televisione nella comunità narrativa italiana-albanese: I grandi occhi del mare di Leonard Guaci, [in:] E. Bond, D. Comberiati (ed.), Il confine liquido. Rapporti letterari e interculturali fra Italia e Albania, Nardò 2013, p. 128.

${ }^{14}$ Ibidem, p. 129.

${ }^{15}$ Ibidem, p. 124.
} 
il suo carattere ludico e conoscitivo. L'arrivo del televisore nelle case albanesi rende la vita più divertente e interessante. Dell'attrezzo approfittano soprattutto i bambini che, attraverso i vari programmi messi loro a disposizione, possono allargare i propri orizzonti, aprirsi verso il mondo per riempirlo di nuove immagini.

La realtà televisiva appare differente, bella e affascinante e perciò tutti i figli dei Cipi, tranne Libero, si sentono in dovere di divulgare lo spirito della libertà tipico dell'Occidente per cambiare l'Albania. Libero, il figlio maggiore dei Cipi, si rivela una spia. Mentre i suoi fratelli sono coinvolti nella lotta contro le autorità, lui compie un'azione completamente opposta: essendo uno dei sostenitori più ferventi del regime fa di tutto per eliminare dalla vita sociale qualsiasi forma di ribellione. Il protagonista non svela ai familiari la sua vera identità per un lungo periodo di tempo. Non riesce però a evitarlo. La famiglia non accetta il suo atteggiamento quasi fanatico verso la legge stabilita, l'atteggiamento che lo porta a perquisire la propria casa e perquisitare i propri fratelli. Libero è così saturo di idee antireazionarie che perde il contatto con la realtà, comincia anzi a odiare la famiglia. Dopo il crollo del regime non gli resta che suicidarsi. Il protagonista si dimostra una figura tragica, la vittima di un sistema oppressivo che lo rende un vero e proprio robot, una macchina volta a eseguire ciecamente gli ordini assegnati. Tratteggiando la figura di Libero, Guaci intende mettere in risalto il carattere distruttivo delle istituzioni comuniste, dell'ideologia che distrugge i nuclei familiari. I fratelli hanno difficoltà nel sopportare la sua presenza, al contempo temono di essere denunciati in quanto conosce i loro segreti, come ad esempio, la passione per la televisione che considera una nuova colonizzazione:

(...) la televisione che voi volete guardare vi farà ridere, vi farà piangere, vi farà divertire, ma non vi racconterà mai niente del genere. State subendo, senza nemmeno accorgervene, una nuova colonizzazione. Perché è così, culturalmente, che si realizzano le colonizzazioni moderne ${ }^{16}$.

Libero, facendo uso della nozione di colonizzazione, si riferisce alla presenza coloniale fascista in Albania nella prima metà del Novecento, volta a soddisfare le ambizioni espanionistiche di Mussolini ${ }^{17}$. Ai suoi occhi la televisione diventa una forma di imprigionamento. Pare opportuno rilevare che Daniele Comberiati, parlando della colonizzazione italiana dell'Albania nella produzione di autori migranti di origine albanese, presenta una visione non molto dissimile da quella di Libero. Lo studioso evidenzia che:

${ }^{16}$ L. Guaci, I grandi occhi del mare, p. 190.

${ }^{17}$ A proposito cfr. R. Morozzo della Rocca, Albania. Le radici della crisi, Milano 1997, pp. 91-94. 


\begin{abstract}
il rapporto fra Italia e Albania ha in questi testi un'importanza predominante: si potrebbe anzi asserire che quella italiana nei confronti del paese balcanico sia stata una doppia colonizzazione, nata in principio con l'invasione del 1939 e proseguita attraverso l'influenza della televisione italiana sull'immaginario collettivo albanese durante gli ultimi anni del comunismo ${ }^{18}$.
\end{abstract}

Il fenomeno può sicuramente essere visto in tale ottica, occorre però tenere presente che la colonizzazione cominciata con l'invasione del 1939 riguarda una campagna militare organizzata contro la volontà degli albanesi, guardare la televisione è invece un'azione compiuta volutamente.

I figli dei Cipi si impegnano in due progetti contro le autorità. Il primo progetto concerne la preparazione della gazzetta „Horizont”, distribuita di nascosto in trenta case scelte a caso in tutta la città, sulle cui pagine si pubblicano le notizie più importanti dal mondo e vari argomenti di natura socioculturale. Il secondo progetto è il gruppo musicale "Suoni Liberi Band". I ragazzi iniziano a dare concerti, prima a casa, poi nei posti pubblici. Il canto è riservato ad Aulona che, grazie alle lezioni d'italiano ottenute da Zyz e alla possibilità di guardare la televisione, riesce a cantare e comunicare bene in questa lingua. Aulona approfitta dell'occasione di praticare la lingua italiana quando per caso s'imbatte in tre italiani giunti a Valona "con una piccola troupe di tecnici sportivi a seguito del grande nuotatore Pinto"19. Gli stranieri si meravigliano del livello della sua competenza linguistica. Le fanno notare che il mondo presentato in televisione può essere diverso da quello reale e la incoraggiano ad andare in Italia. Nella ragazza si sveglia il desiderio di realizzarlo. Insieme al fratello Stalin, e in accordo con i genitori, tentano di attraversare il mare di nascosto in nave. Il progetto non riesce in quanto le navi albanesi, vista la mancanza di rapporti commerciali con il resto del mondo, non giungono sull'altra sponda dell'Adriatico, girano a vuoto e tornano al porto. Così i ragazzi sono costretti a rimanere in Albania. Va precisato che Aulona non potrà recarsi in Italia che dopo il crollo del comunismo.

La musica si presenta come una forma efficace di lotta. Lo si percepisce quando i fratelli Cipi si esibiscono durante la festa della maturità al Liceo Tecnico. Uscito il direttore, un sostenitore strenuo del partito, il gruppo può diffondere l'italianità e lo spirito occidentale fra i maturandi.

Un suono elettrico modello Deep Purple pervase per un attimo l'ambiente. L'attenzione di tutti i giovani venne catturata da quelle note impazzite. (...) I giovani maturandi ebbero qualche secondo di timore, ma quando quella musica

\footnotetext{
${ }^{18} \mathrm{D}$. Comberiati, Riscrivere la storia. Modalità di rappresentazione del colonialismo italiano in Albania, [in:] Incontri. Rivista europea di studi italiani 28, 2013, pp. 28-29.

${ }^{19}$ L. Guaci, I grandi occhi del mare, p. 221.
} 


\begin{abstract}
forte invase la sala non poterono resistere. Abbandonarono le paure e si lasciarono trascinare dal ritmo scatenato. (...) Assaporavano il primo raggio di libertà, la prima soffiata del vento proveniente dall'ovest. Aulona suonava in piedi. (...) Fu anche lei presa dall'euforia generale e non si poteva più fermare. Uno dietro l'altro tirava fuori i brani dal suo repertorio (...). Spaziava dal rock al blues, da Jimi Hendrix ai cantautori italiani. Le sue dita si muovevano con piena maestria sulle corde vibranti come le aveva insegnato il suo maestro Zyz. (...) Gli studenti sfogavano tutta l'energia repressa per decenni dai divieti della rivoluzione ${ }^{20}$.
\end{abstract}

Walter Jackson Ong ${ }^{21}$, paragonando la natura della vista a quella dell'udito, rileva una funzione unificatrice di esso. Il suono è capace di far unire gli individui, influisce sul loro atteggiamento verso la realtà rendendoli fedeli a un ideale preciso. La teoria dello studioso va perfettamente d'accordo con il ruolo che il suono riveste durante la festa della maturità. I giovani si immergono nella musica per costituire un gruppo omogeneo che protesta contro le regole imposte dal sistema. Il progetto dei fratelli Cipi ha il risultato prefissato e non bisogna aspettare a lungo che la loro attività si propaghi in tutta la città di Valona. L'informazione sul concerto giunge alla polizia che, visto il numero dei giovani, non è in grado di punirli. L'arresto dei maturandi avrebbe suscitato la ripugnanza per il regime nei membri di circa cento famiglie. Le autorità si limitano dunque a parole di avvertimento intrise di accenni al carattere degenerativo della musica occidentale. Altri seguono l'esempio dei Cipi e così appaiono nuovi gruppi musicali dalle caratteristiche simili. Il concerto di "Suoni Liberi Band” dà spunto, come dice Guaci ${ }^{22}$, alla nuova rivoluzione che infatti parte dalla musica. Il trapasso di Enver Hoxha porta un soffio di libertà e ne agevola lo sviluppo. La cultura italiana diviene sempre più diffusa. Molti membri della società albanese contrappongono la propria realtà segnata dalla mancanza di mezzi per vivere a quella italiana, cioè ricca e considerata garante di felicità. Aulona e i fratelli, coscienti di tale tendenza, decidono di dare il colpo finale al regime: organizzano una vera e propria ribellione, ossia il primo concerto rock "Jeans contro la Rivoluzione”. Il nome del concerto è simbolico ed esprime il desiderio di libertà provato dagli albanesi che attendono con impazienza il giorno in cui potranno portare i jeans, „sintomo di una vera libertà" ${ }^{2}$. L'evento a cui partecipano anche le altre nuove band anticomuniste, cosparso di sangue di numerose persone, contribuisce al crollo definitivo del sistema e rende vincitori tutti gli amanti della cultura italiana, costretti per molti anni a camuffare la loro passione.

\footnotetext{
${ }^{20}$ Ibidem, p. 202.

${ }^{21}$ W.J. Ong, Oralità e scrittura, p. 106.

${ }^{22}$ L. Guaci, I grandi occhi del mare, p. 204.

${ }^{23}$ Ibidem, p. 263.
} 
Pare interessante rilevare che un ampio quadro dell'Albania di fine dell'epoca del comunismo è delineato nel romanzo di Ron Kubati (1971) "Va e non torna” (2000). Lo spunto per lottare contro le autorità comuniste viene dato, come in Guaci, dai partecipanti di un evento sociale. A Kavaja, una piccola località vicino a Durazzo, ,dopo una partita di calcio, la protesta contro gli errori dell'arbitro era diventata rivolta aperta” e la città „era diventata quasi libera" 24 . Sulla scia dell'avvenimento vengono organizzate delle manifestazioni miranti a esprimere il disappunto verso le condizioni di vita tipiche del paese, a eliminare qualsiasi presenza del defunto Enver Hoxha. Siccome la sua statua rimane sempre nella piazza centrale della capitale, molti personaggi decidono di rimuoverla perché la caduta della figura del dittatore simboleggia la caduta dello stesso regime. Particolarmente impegnati nelle iniziative reazionarie sono gli studenti desiderosi di cambiamenti e di nuove possibilità. I giovani, come ne „I grandi occhi del mare”, sono considerati un gruppo molto importante, dotato di una forza creatrice capace di dare vita a un nuovo mondo che garantirà la libertà e forse consentirà loro di recarsi altrove ${ }^{25}$. In un suo saggio, dedicato tra l'altro al romanzo „Va e non torna”, lo stesso Kubati sottolinea che:

(...) la cultura del giovane, la cultura dell'immigrato, della potenza dell'assenza, di ogni nuovo inizio, è la cultura del desiderio, è la cultura dell'anelito il cui orizzonte è l'altrimenti, il cui orizzonte è l'altrove ${ }^{26}$.

\section{LA NUOVA IDENTITÀ}

$\mathrm{Ne}$ „I luoghi della cultura” Homi Bhabha ${ }^{27}$ fa uso della nozione di spazi „inter-mediari”; sono luoghi egemonici portatori di una cultura che caratterizza un'istanza di mediazione. Questi spazi „costituiscono il terreno per l'elaborazione di strategie del sé - come singoli o gruppo - che danno il via a nuovi segni di identità e luoghi innovativi in cui sviluppare la collaborazione e la contestazione nell'atto stesso in cui si definisce l'idea di società" ${ }^{28}$. Per Cristofaro Longo ${ }^{29}$ la mediazione culturale significa „l'operazione culturale

\footnotetext{
${ }^{24}$ R. Kubati, Va e non torna, Nardò 2000, p. 141.

${ }^{25}$ A proposito si veda S. Wright, „Va e non torna” e „M”, ovvero la poetica dell'altrove e dell'altrimenti nei romanzi di Ron Kubati, NEMLA Italian Studies 27-28, 2003-2004, pp. 113-133.

${ }^{26}$ R. Kubati, Alla ricerca dell'altrimenti „Va e non torna” e „M”, [in:] A. Gnisci (ed.), Allattati dalla lupa. Scritture migranti, Roma 2005, p. 53.

${ }^{27}$ H.K. Bhabha, I luoghi della cultura, Roma 2001, p. 12.

${ }^{28}$ Ibidem.

${ }^{29}$ G. Di Cristofaro Longo, Identità e cultura, p. 31.
} 
conseguente all'incontro, storicamente determinato, del soggetto con la sua cultura di riferimento". Nel romanzo di Guaci confluiscono ambedue le definizioni. Seguendole, possiamo constatare che l'Italia è un vero e proprio spazio inter-mediario. La cultura italiana, come detto, influisce in modo enorme sull'immaginario degli albanesi e la sua presenza in Albania risulta tra l'altro da implicazioni storiche. La cultura, pertinente al luogo egemonico, viene accettata da individui provenienti da un paese meno importante legato a esso dal passato coloniale. Abbiamo dunque a che fare con la costruzione di una dimensione innovativa in cui l'identità minoritaria, al contrario di quanto accade sovente nei testi di autori postcoloniali, non sente il bisogno di sottolineare la propria diversità. Vista la specificità dei condizionamenti politici e economici dell'Albania comunista, essa adotta la cultura italiana per trattarla come propria. Si compie così una particolare operazione transnazionale che porta a un rinsaldamento identitario e culturale. „Il processo di traduzione/ /trasposizione culturale" ${ }^{30}$ ha un significato pluridimensionale: forgia non solo l'identità ma anche l'atteggiamento verso il mondo circostante. L'individuo non abbandona il paese d'origine per identificarsi con la cultura dell'altro. Tale processo avviene proprio in terra natale e ciò, occorre dirlo, costituisce un tratto distintivo del romanzo in quanto la tecnica più frequente nei cosiddetti migrant writers d'espressione italiana è quella di trattare il tema dell'identità dopo l'arrivo del protagonista nel paese ospitante ${ }^{31}$.

Come nota Dominique Chancés ${ }^{2}$, l'incontro con l'alterità influisce enormemente sull'identità, spinge alla sua ricerca. L'immagine dell'altro ammirata alla televisione si dimostra una forza creatrice che affascina e stimola la ricerca

${ }^{30}$ H.K. Bhabha, I luoghi della cultura, p. 239.

${ }^{31}$ Come esempio possiamo addurre il romanzo di Artur Spanjolli intitolato „Eduart” (2005). Il protagonista (il suo nome costituisce il titolo dell'opera), un giovane albanese intraprende un viaggio in Italia dove inizialmente è costretto a fronteggiare vari problemi: da quelli socioeconomici a quelli identitari. Eduart ha difficoltà nell'ambientarsi nella cultura dell'altro, ma dopo qualche tempo vi si abitua. L'alterità si dimostra ostile, provoca solitudine e un senso di spaesamento alla protagonista dell'opera di Geneviève Makaping "Traiettorie di sguardi. E se gli altri foste voi?" (2001), una camerunese che vive in Italia. Incontrare l'altro, significa provare ostilità, respingimento. La donna rileva come per gli italiani sia peggiore: in treno evitano il posto libero accanto a lei, la attacano con espressioni moleste, in genere risultano razzisti pieni di pregiudizi nei confronti degli stranieri. A proposito del tema del razzismo nella letteratura italiana contemporanea si veda il libro di Riccardo Bonavita „Spettri dell'altro. Letteratura e razzismo nell'Italia contemporanea”, Bologna 2009. Sull'esclusione dei migranti nel paese d'arrivo si consulti l'opera di Alessandro Dal Lago „Non-persone. L'esclusione dei migranti in una società globale”, Milano 1999.

${ }^{32}$ D. Chancé, Altérité, [in:] M. Beniamino, L. Gauvin (ed.), Vocabulaire des études francophones, Limoges 2005, p. 18. 
identitaria. La sua influenza è così intensa che gli stessi personaggi principali diventano altri, cioè portatori di cultura italiana.

Nel romanzo di Guaci, conformemente alla teoria di Hannah Arendt ${ }^{33}$, l'identità risulta performativa, cioè mutabile; è un concetto che subisce trasformazioni inerenti alle esperienze vissute, alle informazioni acquisite nel cammino esistenziale. In quest'ottica lo scrittore conserva il paradigma che caratterizza il motivo dell'identità diffuso in altri scrittori migranti d'espressione italiana e origine albanese ${ }^{34}$. Va aggiunto però che nella loro produzione di solito l'appartenenza identitaria si modifica in seguito a un viaggio, all'arrivo dell'individuo in terra straniera. Nell'opera in esame invece, come visto, l'identità si costruisce in Albania attraverso la scoperta di una nuova cultura, tanto diversa da quella albanese. Possiamo azzardare l'ipotesi che i protagonisti del romanzo subiscano il processo di acculturazione che usualmente avviene nelle cosiddette società postcoloniali. Non si tratta però di un processo imposto con violenza dal colonizzatore, al contrario, l'acquisizione della nuova identità culturale procede in accordo con la volontà dell'individuo stanco della vita nella realtà opprimente del proprio paese e intenzionato ad aprirsi verso il nuovo mondo e i suoi valori. Giovanni Marchetti nota che:

L'esistenza, quasi sempre conflittiva, di etnie diverse alimenta e rende non di rado tormentato il processo di definizione di nuove 'identità': identità culturali che si propongono, talora come unificanti, costruite attorno agli eventi della nuova storia della comunità, miticamente trasfigurati e conciliati con quelli di una storia antica, riplasmata ad arte; o che invece propendono per la divaricazione e il recupero, talvolta anacronistico e ugualmente fuorviante, delle tradizioni "native"35.

Nell'ottica delle parole di Marchetti il comunismo diventa il colonizzatore che impone il proprio modello culturale ${ }^{36}$ e così limita la libertà dell'individuo, o meglio, fa di tutto per renderlo ubbidiente. Molti personaggi si oppongono in quanto, come detto, sono attratti dalla cultura dell'altro,

\footnotetext{
${ }^{33}$ H. Arendt, The Human Condition, Chicago-London 1958, p. 193.

${ }^{34}$ Nell'opera "La mano che non mordi” (2007) di Ornela Vorpsi (1968) la protagonista, un'albanese che vive in Francia, affronta una problematica situazione identitaria; risulta difficile determinare precisamente la sua appartenenza culturale e nazionale. Identificarsi con la cultura balcanica e albanese, con la terra di origine in generale si dimostra impossibile visto tra l'altro il legame con l'Occidente. Abbiamo a che fare con un'identità fluida che subisce modificazioni inerenti alle esperienze di vita.

${ }^{35}$ G. Marchetti, Lingua/Cultura, [in:] S. Albertazzi, R. Vecchi (ed.), Abbecedario postcoloniale, Macerata 2001, p. 53.

${ }^{36}$ Alcuni elementi della cultura propagata dal regime comunista vengono tratteggiati nell'opera di Anilda Ibrahimi (1972) „Rosso come una sposa” (2008). A proposito si veda anche: „Paese dove non si muore mai" (2005) di Ornela Vorpsi.
} 
dalla cultura italiana. Abbiamo a che fare con un rovesciamento totale della relazione usuale fra colonizzato e colonizzatore diffusa nelle opere degli autori postcoloniali. In Guaci il comunismo diventa, come detto, colonizzatore che imprigiona l'individuo. Si tratta dunque di un colonizzatore interno e non di un'oppressione straniera. Il processo di colonizzazione avviene all'interno di un paese, di una nazione. Il colonizzato cerca una via di scampo, una meta esterna con cui potrà identificarsi e paradossalmente la trova dall'altra parte dell'Adriatico. L'Italia, storicamente il colonizzatore, da una prospettiva culturale gli pare più amichevole dell'Albania. Con il crollo del regime il paese sarà pronto a costruire i pilastri di una nuova cultura che rifletta le aspettative dei cittadini. In questo contesto la cultura, similmente all'identità, risulta un concetto che si evolve; come sostiene Gayatry Spivak, „la cultura è incessantemente in movimento, produce i suoi controesempi, soggetti alla (non) legge della differenza" ${ }^{37}$.

\section{CONCLUSIONI}

La cultura italiana in Albania, sebbene sia continuamente negata e soffocata dalle autorità comuniste, acquisisce il ruolo di un vero e proprio catalizzatore che trasforma la mentalità anacronistica di molti albanesi per forgiare la loro identità, prima in una prospettiva micro, cioè limitata a pochi individui, poi in quella macro che ingloba le masse capaci di manifestare la propria forza e raggiungere lo scopo prefissato. Il fascino dell'italianità e l'odio del comunismo fanno sì che numerose persone inizino a indentificarsi con la cultura dell'altro, che paradossalmente è più vicina alla loro autocoscienza, al loro senso di giustizia, alla loro umanità. Possiamo parlare della costruzione della nuova identità culturale che riflette il bisogno di ogni essere umano di conservare la calma interiore, di vivere in armonia con se stesso e, se adottiamo un punto di vista più largo, di raggiungere la felicità. Da un lato Guaci, mostrando l'individuo in uno specifico contesto conflittuale di respiro universale, trasmette un messaggio di matrice antropologica, dall'altro pone l'accento sul legame inseparabile delle due sponde dell'Adriatico: quella italiana e quella albanese.

${ }^{37}$ G. Spivak, Perché il pianeta? Un'autobiografia intellettuale, [in:] S. Adamo (ed.), Culture planetarie? Prospettive e limiti della teoria e della critica culturale, Roma 2007, p. 47. 


\section{Karol Karp \\ KONFLIKT MIĘDZY JEDNOSTKĄ A SYSTEMEM: W KIERUNKU UKSZTAŁTOWANIA SIĘ NOWEJ TOŻSAMOŚCI KULTUROWEJ W POWIEŚCI „I GRANDI OCCHI DEL MARE” LEONARDA GUACIEGO}

\section{Streszczenie}

Artykuł analizuje powieść „I grandi occhi del mare” (2005) Leonarda Guaciego jednego z najbardziej znanych przedstawicieli włoskiej literatury migracyjnej albańskiego pochodzenia. Analiza ma na celu ukazanie zjawiska tożsamości kulturowej. Zależność między tożsamością a kulturą została określona na podstawie teorii Gioi di Cristofaro Longo. Wśród innych badaczy, których ustalenia wykorzystano w pracy, należy wymienić na przykład: Hannah Arendt, Homiego Bhabhę, Giovanniego Marchettiego, Dominique’a Chancé. Kształtowanie się tożsamości kulturowej bohaterów ma związek z ich sprzeciwem wobec zasad, które narzucił im komunistyczny reżim. Pierwsza część artykułu jest poświęcona postaci Zyza - Albańczyka, który w młodości spędził kilka lat we Włoszech i dogłębnie poznał ten kraj. Zostaje tu podkreślona przede wszystkim działalność bohatera zmierzająca do upowszechnienia kultury włoskiej w Albanii. Druga część artykułu dotyczy kulturotwórczej roli mediów i określa ich wpływ na światopogląd Albańczyków. Ostatnia część pracy prezentuje najważniejsze cechy fenomenu tożsamości kulturowej.

\section{Bibliografia}

Arendt H., The Human Condition, Chicago-London 1958.

Bhabha H., I luoghi della cultura, Roma 2001.

Bonavita R., Spettri dell'altro. Letteratura e razzismo nell'Italia contemporanea, Bologna 2009.

Chancé D., Altérité, [in:] M. Beniamino, L. Gauvin (ed.), Vocabulaire des études francophones, Limoges 2005, pp. 17-19.

Comberiati D., Riscrivere la storia. Modalità di rappresentazione del colonialismo italiano in Albania, Incontri. Rivista europea di studi italiani 28, 2013, pp. 25-33.

Dal Lago A., Non-persone. L'esclusione dei migranti in una società globale, Milano 1999.

Di Cristofaro Longo G., Identità e cultura. Per un'antropologia della reciprocità, Roma 1993.

Guaci L., I grandi occhi del mare, Nardò 2005.

Ibrahimi A., Rosso come una sposa, Torino 2008.

Kubati R., Alla ricerca dell'altrimenti „Va e non torna” e „M”, [in:] A. Gnisci (ed.), Allattati dalla lupa. Scritture migranti. Roma 2005, pp. 49-65.

Kubati R., Va e non torna, Nardò 2000.

Makaping G., Traiettorie di sguardi. E se gli altri foste voi?, Soveria Mannelli 2001.

Marchetti G., Lingua/Cultura, [in:] S. Albertazzi, R. Vecchi (ed.), Abbecedario postcoloniale. Macerata 2001, pp. 53-60. 
Moll N., Il ruolo della televisione nella comunità narrativa italiana-albanese: I grandi occhi del mare di Leonard Guaci, [in:] E. Bond, D. Comberiati (ed.), Il confine liquido. Rapporti letterari e interculturali fra Italia e Albania, Nardò 2013, pp. 117-136.

Morozzo della Rocca R., Albania. Le radici della crisi, Milano 1997.

Ong W., Oralità e scrittura. La tecnologia della parola, Bologna 2006.

Ricoeur P., Filozofia osoby, Kraków 1992.

Spanjolli A., I nipoti di Scanderbeg, Nardò 2012.

Spanjolli A., Eduart, Nardò 2005.

Spanjolli A., Cronaca di una vita in silenzio, Nardò, 2003.

Spivak G., Perché il pianeta? Un'autobiografia intellettuale, [in:] S. Adamo (ed.), Culture planetarie? Prospettive e limiti della teoria e della critica culturale, Roma 2007, pp. 41-58.

Vorpsi O., La mano che non mordi, Torino 2007.

Vorpsi O., Il paese dove non si muore mai, Torino 2005.

Wright S., „Va e non torna” e „M”, ovvero la poetica dell'altrove e dell'altrimenti nei romanzi di Ron Kubati, [in:] NEMLA Italian Studies 27-28, 2003-2004, pp. 113-133. 